\title{
Star Physics and Measurement Data
}

\author{
Boris V. Vasiliev \\ Independent Researcher, Dubna, Russia \\ Email:bv.vasiliev@yandex.com
}

How to cite this paper: Vasiliev, B.V. (2018) Star Physics and Measurement Data. Journal of Modern Physics, 9, 1906-1934. https://doi.org/10.4236/jmp.2018.910120

Received: May 28, 2018

Accepted: August 31, 2018

Published: September 3, 2018

Copyright $\odot 2018$ by author and Scientific Research Publishing Inc. This work is licensed under the Creative Commons Attribution International License (CC BY 4.0).

http://creativecommons.org/licenses/by/4.0/

\begin{abstract}
Astrophysics $=$ the star physics was beginning its development without a supporting of measurement data, which could not be obtained then. Still astrophysics exists without this support, although now astronomers collected a lot of valuable information. This is the main difference of astrophysics from all other branches of physics, for which foundations are measurement data. The creation of the theory of stars, which is based on the astronomical measurements data, is one of the main goals of modern astrophysics. Below, the principal elements of star physics based on data of astronomical measurements are described. The theoretical description of a hot star interior is obtained. It explains the distribution of stars over their masses, mass-radius-temperature and mass-luminosity dependencies. All theoretical predictions are in a good agreement with the known measurement data, which confirms the validity of this consideration.
\end{abstract}

\section{Keywords}

Electric Polarization, Plasma, Stellar Mass, Stellar Temperature, Stellar Radius

\section{Introduction}

\subsection{Astrophysics and Astronomical Measurements}

It is obvious that the primary goal of modern astrophysics is to create a star theory that can explain the dependencies of parameters of stars and of the Sun, which are measured by astronomers.

The technical progress of astronomical measurements in the last decade has revealed the existence of different relationships that associate together the physical parameters of the stars.

To date, such data have accumulated about a dozen: they are dependencies of temperature-radius-luminosity-mass of stars, the spectra of seismic oscillations of the sun, distribution of stars on mass, the dependence of the magnetic fields 
of stars from their moments and speeds of rotation, etc.

All these relationships are defined by phenomena occurring inside stars. So the theory of the internal structure of stars should be based on these quantitative data as on boundary conditions.

Existing theories of stellar interiors can not explain the new data. The modern astrophysics ${ }^{1}$ prefers a speculative approach. It elaborates qualitative theories of stars that are not pursued to such quantitative estimates, which could be compared with the data of astronomers. Everything is done in such a way as if the new astronomical data are absent. Of course, the astrophysical community knows about the existence of dependencies of stellar parameters which were measured by astronomers. However, in modern astrophysics, it is accepted to think, that if an explanation of a dependency is not found, that it can be referred to the category of empirical one and it needs no explanation. The so-called empirical relations of stellar luminosities and temperatures-the Hertzsprung-Russell diagram - are known about the hundred years, but its quantitative explanation is not found.

The reason that prevents to explain these relationships is due to the wrong choice of the basic postulates of modern astrophysics. Despite of the fact that all modern astrophysics believes that the stars consist from a plasma, it historically turned out that the theory of stellar interiors does not take into account the electric polarization of the plasma, which must occur within stars under the influence of their gravitational field. Modern astrophysics believes that the gravity-induced electric polarization (GIEP) of stellar plasma is small and it should not be taken into account in the calculations, as this polarization was not taken into account in the calculations at an early stage of development of astrophysics, when a plasma structure of stars was not known. However, plasma is an electrically polarized substance, and an exclusion of the GIEP effect from the calculation is unwarranted. Moreover, without taking into account of the GIEP-effect, the equilibrium stellar matter can not be correctly founded and a theory would not be able to explain the astronomical measurements. Accounting GIEP gives the theoretical explanation for the all observed dependence.

As shown below, the account of the gravity-induced electric polarization of the intra-stellar plasma gives possibility to develop a model of the star, in which all main parameters-the mass of the star, its temperature, radius and luminosity-are expressed by certain combinations of world constants and the individuality of stars is determined by only two parameters-the mass and charge number of nuclei, from which the plasma of these stars is composed. It gives the quantitatively and fairly accurate explanation of all dependencies, which were measured by astronomers.

The important feature of this stellar theory, which is built with the GIEP

${ }^{1}$ The modern astrophysics has a whole series of different branches. It is important to stress that all of them except the physics of hot stars beyond the scope of this consideration; we shall use the term "astrophysics" here and below in its initial meaning-as the physics of stars. 
acconting, is the lack of a collapse in the final stage of the star development, as well as "black holes" that could be results from a such collapse.

The main features of this concept were previously published in [1]-[6].

\subsection{The Basic Postulate of Astrophysics}

We can assume that modern astrophysics emerged in the early twentieth century and milestone of this period was the work R. Emden 《Die Gaskugeln》. It has laid the basis for the description of stars as gas spheres. Gases can be characterized by different dependencies of their density from pressure, ie they can be described by different polytropes. According to Emden the equations of state of the gases producing the stars determine their characteristics-it can be either a dwarf, or a giant, or main sequence star, etc. The such approach to the description of stars determined the choice of postulates needed for the theory.

Any theory based on its system of postulates.

The first basic postulate of astrophysics - the Euler equation - was formulated in a mathematical form by L.Euler in a middle of 18th century for the "terrestrial" effects description. This equation determines the equilibrium condition of liquids or gases in a gravitational field:

$$
\gamma \boldsymbol{g}=-\nabla P .
$$

According to it the action of a gravity forth $\gamma \boldsymbol{g}$ ( $\gamma$ is density of substance, $\boldsymbol{g}$ is the gravity acceleration) in equilibrium is balanced by a forth which is induced by the pressure gradient in the substance.

All modern models of stellar interior are obtained on the base of the Euler equation. These models assume that pressure inside a star monotone increases depthward from the star surface. As a star interior substance can be considered as an ideal gas which pressure is proportional to its temperature and density, all astrophysical models predict more or less monotonous increasing of temperature and density of the star substance in the direction of the center of a star.

While we are talking about materials with atomic structure, there are no doubt about the validity of this equation and its applicability. This postulate is surely established and experimentally checked be "terrestrial" physics. It is the base of an operating of series of technical devices-balloons, bathyscaphes and other.

Another prominent astrophysicist first half of the twentieth century was A. Eddington. At this time I. Langmuir discovered the new state of matter-plasma. A. Eddington was first who realized the significance of this discovery for astrophysics. He showed that the stellar matter at the typical pressures and temperatures, should be in the plasma state.

The polarizability of atomic matter is negligible. ${ }^{2}$ There was not necessary to take into account a polarization while there was a speech about the model, in ${ }^{2}$ If you do not take account of ferroelectrics, piezoelectrics and other similar substances. Their consideration is not acceptable here. 
which the stars are composed of atomic gases.

But plasma is an electrically polarized substance.

\section{It Is Necessary to Take into Account GIEP of Intra-Stellar Plasma}

Therefore, at consideration of a plasma equilibrium, the term describing its possible electrical polarization $\mathfrak{P}$ should be saved in the Euler equation:

$$
\gamma \boldsymbol{g}+\mathfrak{P} \nabla \mathfrak{P}+\nabla P=0,
$$

This leads to the possibility of the existence of a fundamentally new equilibrium state of stellar matter, at which it has a constant density and temperature:

$$
\begin{gathered}
\nabla P=0 \\
\gamma \boldsymbol{g}+\mathfrak{P} \nabla \mathfrak{P}=0,
\end{gathered}
$$

that radically distinguishes this equilibrium state from equilibrium, which is described by Equation (1).

The technical progress of astronomical measurements in the last decade discovered that the physical parameters of the stars are related together.

However, these new data do not fit to models of modern astrophysics.

\section{The Energy-Favorable State of Hot Dense Plasma}

\subsection{The Properties of a Hot Dense Plasma}

\subsubsection{A Hot Plasma and Boltzman's Distribution}

Free electrons being fermions obey the Fermi-Dirac statistic at low temperatures. At high temperatures, quantum distinctions in behavior of electron gas disappear and it is possible to consider electron gas as the ideal gas which obeys the Boltzmann's statistics. At high temperatures and high densities, all substances transform into electron-nuclear plasma. There are two tendencies in this case. At temperature much higher than the Fermi temperature $T_{F}=\frac{\mathcal{E}_{F}}{k}$ (where $\mathcal{E}_{F}$ is Fermi energy), the role of quantum effects is small. But their role grows with increasing of the pressure and density of an electron gas. When quantum distinctions are small, it is possible to describe the plasma electron gas as a the ideal one. The criterium of Boltzman's statistics applicability

$$
T \gg \frac{\mathcal{E}_{F}}{k} \text {. }
$$

hold true for a non-relativistic electron gas with density $10^{25}$ particles in $\mathrm{cm}^{3}$ at $T \gg 10^{6} \mathrm{~K}$.

At this temperatures, a plasma has energy

$$
\mathcal{E}=\frac{3}{2} k T N
$$

and its EOS is the ideal gas EOS:

$$
P=\frac{N k T}{V}
$$


But even at so high temperatures, an electron-nuclear plasma can be considered as ideal gas in the first approximation only. For more accurate description its properties, the specificity of the plasma particle interaction must be taken into account and two main corrections to ideal gas law must be introduced.

The first correction takes into account the quantum character of electrons, which obey the Pauli principle, and cannot occupy levels of energetic distribution which are already occupied by other electrons. This correction must be positive because it leads to an increased gas incompressibility.

Other correction takes into account the correlation of the screening action of charged particles inside dense plasma. It is the so-called correlational correction. Inside a dense plasma, the charged particles screen the fields of other charged particles. It leads to a decreasing of the pressure of charged particles. Accordingly, the correction for the correlation of charged particles must be negative, because it increases the compressibility of electron gas.

\subsubsection{The Hot Plasma Energy with Taking into Account the Correction for the Fermi-Statistic}

The energy of the electron gas in the Boltzmann's case $\left(k T \gg \mathcal{E}_{F}\right)$ can be calculated using the expression of the full energy of a non-relativistic Fermi-particle system [7]:

$$
\mathcal{E}=\frac{2^{1 / 2} V m_{e}^{3 / 2}}{\pi^{2} \hbar^{3}} \int_{0}^{\infty} \frac{\varepsilon^{3 / 2} \mathrm{~d} \varepsilon}{e^{\left(\varepsilon-\mu_{e}\right) / k T}+1}
$$

expanding it in a series. ( $m_{e}$ is electron mass, $\varepsilon$ is the energy of electron and $\mu_{e}$ is its chemical potential).

In the Boltzmann's case, $\mu_{e}<0$ and

$\left|\mu_{e} / k T\right| \gg 1$ and the integrand at

$e^{\mu_{e} / k T} \ll 1$ can be expanded into a series according to powers $e^{\mu_{e} / k T-\varepsilon / k T}$. If

we introduce the notation $z=\frac{\varepsilon}{k T}$ and conserve the two first terms of the series, we obtain

$$
I \equiv(k T)^{5 / 2} \int_{0}^{\infty} \frac{z^{3 / 2} \mathrm{~d} z}{e^{z-\mu_{e} / k T}+1} \approx(k T)^{5 / 2} \int_{0}^{\infty} z^{3 / 2}\left(e^{\frac{\mu_{e}}{k T}-z}-e^{2\left(\frac{\mu_{e}}{k T}-z\right)}+\cdots\right) \mathrm{d} z
$$

or

$$
\frac{I}{(k T)^{5 / 2}} \approx e^{\frac{\mu_{e}}{k T}} \Gamma\left(\frac{3}{2}+1\right)-\frac{1}{2^{5 / 2}} e^{\frac{2 \mu_{e}}{k T}} \Gamma\left(\frac{3}{2}+1\right) \approx \frac{3 \sqrt{\pi}}{4} e^{\mu_{e} / k T}\left(1-\frac{1}{2^{5 / 2}} e^{\mu_{e} / k T}\right) .
$$

Thus, the full energy of the hot electron gas is

$$
\mathcal{E} \approx \frac{3 V}{2} \frac{(k T)^{5 / 2}}{\sqrt{2}}\left(\frac{m_{e}}{\pi \hbar^{2}}\right)^{3 / 2}\left(e^{\mu_{e} / k T}-\frac{1}{2^{5 / 2}} e^{2 \mu_{e} / k T}\right) .
$$

Using the definition of a chemical potential of ideal gas (of particles with spin $=1 / 2)[7]$ 


$$
\mu_{e}=k T \log \left[\frac{N_{e}}{2 V}\left(\frac{2 \pi \hbar^{2}}{m_{e} k T}\right)^{3 / 2}\right]
$$

we obtain the full energy of the hot electron gas

$$
\mathcal{E}_{e} \approx \frac{3}{2} k T N_{e}\left[1+\frac{\pi^{3 / 2}}{4}\left(\frac{a_{B} e^{2}}{k T}\right)^{3 / 2} n_{e}\right],
$$

where $a_{B}=\frac{\hbar^{2}}{m_{e} e^{2}}$ is the Bohr radius.

\subsubsection{The Correction for Correlation of Charged Particles in a Hot Plasma}

At high temperatures, the plasma particles are uniformly distributed in space. At this limit, the energy of ion-electron interaction tends to zero. Some correlation in space distribution of particles arises as the positively charged particle groups around itself preferably particles with negative charges and vice versa. It is accepted to estimate the energy of this correlation by the method developed by Debye-Hükkel for strong electrolytes [7]. The energy of a charged particle inside plasma is equal to $e \varphi$, where $e$ is the charge of a particle, and $\varphi$ is the electric potential induced by other particles on the considered particle.

This potential inside plasma is determined by the Debye law [7]:

$$
\varphi(r)=\frac{e}{r} e^{-\frac{r}{r_{D}}}
$$

where the Debye radius is

$$
r_{D}=\left(\frac{4 \pi e^{2}}{k T} \sum_{a} n_{a} Z_{a}^{2}\right)^{-1 / 2}
$$

For small values of ratio $\frac{r}{r_{D}}$, the potential can be expanded into a series

$$
\varphi(r)=\frac{e}{r}-\frac{e}{r_{D}}+\cdots
$$

The following terms are converted into zero at $r \rightarrow 0$. The first term of this series is the potential of the considered particle. The second term

$$
\mathcal{E}=-e^{3} \sqrt{\frac{\pi}{k T V}}\left(\sum_{a} N_{a} Z_{a}^{2}\right)^{3 / 2}
$$

is a potential induced by other particles of plasma on the charge under consideration. And so the correlation energy of plasma consisting of $N_{e}$ electrons and ( $N_{e} / Z$ ) nuclei with charge $Z$ in volume $V$ is [7]

$$
\mathcal{E}_{\text {corr }}=-e^{3} \sqrt{\frac{\pi n_{e}}{k T}} Z^{3 / 2} N_{e}
$$




\subsection{The Energy-Preferable State of a Hot Plasma}

\subsubsection{The Energy-Preferable Density of a Hot Plasma}

Finally, under consideration of both main corrections taking into account the inter-particle interaction, the full energy of plasma is given by

$$
\mathcal{E} \approx \frac{3}{2} k T N_{e}\left[1+\frac{\pi^{3 / 2}}{4}\left(\frac{a_{B} e^{2}}{k T}\right)^{3 / 2} n_{e}-\frac{2 \pi^{1 / 2}}{3}\left(\frac{Z}{k T}\right)^{3 / 2} e^{3} n_{e}^{1 / 2}\right]
$$

The plasma into a star has a feature. A star generates the energy into its inner region and radiates it from the surface. At the steady state of a star, its substance must be in the equilibrium state with a minimum of its energy. The radiation is not in equilibrium of course and can be considered as a star environment. The equilibrium state of a body in an environment is related to the minimum of the function $([7] \S 20)$ :

$$
\mathcal{E}-T_{o} S+P_{o} V
$$

where $T_{o}$ and $P_{o}$ are the temperature and the pressure of an environment. At taking in to account that the star radiation is going away into vacuum, the two last items can be neglected and one can obtain the equilibrium equation of a star substance as the minimum of its full energy:

$$
\frac{\mathrm{d} \mathcal{E}_{\text {plasma }}}{\mathrm{d} n_{e}}=0 .
$$

Now taking into account Equation (18), one obtains that an equilibrium condition corresponds to the equilibrium density of the electron gas of a hot plasma

$$
n_{e}^{\text {equilibrium }} \equiv n_{\star}=\frac{16}{9 \pi} \frac{Z^{3}}{a_{B}^{3}} \approx 1.2 \times 10^{24} Z^{3} \mathrm{~cm}^{-3},
$$

It gives the electron density $\approx 3 \times 10^{25} \mathrm{~cm}^{-3}$ for the equilibrium state of the hot plasma of helium.

\subsubsection{The Estimation of Temperature of Energy-Preferable State of a Hot Stellar Plasma}

As the steady-state value of the density of a hot non-relativistic plasma is known, we can obtain an energy-preferable temperature of a hot non-relativistic plasma.

The virial theorem [7] [8] claims that the full energy of particles $E$, if they form a stable system with the Coulomb law interaction, must be equal to their kinetic energy $T$ with a negative sign. Neglecting small corrections at a high temperature, one can write the full energy of a hot dense plasma as

$$
\mathcal{E}_{\text {plasma }}=U+\frac{3}{2} k T N_{e}=-\frac{3}{2} k T N_{e} .
$$

where $U \approx-\frac{G \mathbb{M}^{2}}{\mathbb{R}_{0}}$ is the potential energy of the system, $G$ is the gravitational constant, $\mathbb{M}$ and $\mathbb{R}_{0}$ are the mass and the radius of the star.

As the plasma temperature is high enough, the energy of the black radiation 
cannot be neglected. The full energy of the stellar plasma depending on the particle energy and the black radiation energy

$$
\mathcal{E}_{\text {total }}=-\frac{3}{2} k T N_{e}+\frac{\pi^{2}}{15}\left(\frac{k T}{\hbar c}\right)^{3} V k T
$$

at equilibrium state must be minimal, i.e.

$$
\left(\frac{\partial \mathcal{E}_{\text {total }}}{\partial T}\right)_{N, V}=0
$$

This condition at $\frac{N_{e}}{V}=n_{\star}$ gives a possibility to estimate the temperature of the hot stellar plasma at the steady state:

$$
\mathbb{T}_{\star} \approx Z \frac{\hbar c}{k a_{B}} \approx 10^{7} Z \mathrm{~K} .
$$

The last obtained estimation can raise doubts. At "terrestrial" conditions, the energy of any substance reduces to a minimum at $T \rightarrow 0$. It is caused by a positivity of a heat capacity of all of substances. But the steady-state energy of star is negative and its absolute value increases with increasing of temperature (Equation (22)). It is the main property of a star as a thermodynamical object. This effect is a reflection of an influence of the gravitation on a stellar substance and is characterized by a negative effective heat capacity. The own heat capacity of a stellar substance (without gravitation) stays positive. With the increasing of the temperature, the role of the black radiation increases $\left(\mathcal{E}_{b r} \sim T^{4}\right)$. When its role dominates, the star obtains a positive heat capacity. The energy minimum corresponds to a point between these two branches.

\section{The Gravity Induced Electric Polarization in a Dense Hot Plasma}

\subsection{Plasma Cells}

The existence of plasma at energetically favorable state with the constant density $n_{\star}$ and the constant temperature $\mathbb{T}_{\star}$ puts a question about equilibrium of this plasma in a gravity field. The Euler equation in commonly accepted form Equation (1) disclaims a possibility to reach the equilibrium in a gravity field at a constant pressure in the substance: the gravity inevitably must induce a pressure gradient into gravitating matter. To solve this problem, it is necessary to consider the equilibrium of a dense plasma in an gravity field in detail. At zero approximation, at a very high temperature, plasma can be considered as a "jelly", where electrons and nuclei are "smeared" over a volume. At a lower temperature and a high density, when an interpartical interaction cannot be neglected, it is accepted to consider a plasma dividing in cells [9]. Nuclei are placed at centers of these cells, the rest of their volume is filled by electron gas. Its density decreases from the center of a cell to its periphery. Of course, this dividing is not freezed. Under action of heat processes, nuclei move. But having a small mass, electrons have time to trace this moving and to form a permanent electron cloud around 
nucleus, i.e. to form a cell. So plasma under action of a gravity must be characterized by two equilibrium conditions:

- the condition of an equilibrium of the heavy nucleus inside a plasma cell;

- the condition of an equilibrium of the electron gas, or equilibrium of cells.

\subsection{The Equilibrium of a Nucleus Inside Plasma Cell Filled by an Electron Gas}

At the absence of gravity, the negative charge of an electron cloud inside a cell exactly balances the positive charge of the nucleus at its center. Each cell is fully electroneutral. There is no direct interaction between nuclei.

The gravity acts on electrons and nuclei at the same time. Since the mass of nuclei is large, the gravity force applied to them is much larger than the force applied to electrons. On the another hand, as nuclei have no direct interaction, the elastic properties of plasma are depending on the electron gas reaction. Thus er have a situation, when the force applied to nuclei must be balanced by the force of the electron subsystem. The cell obtains an electric dipole moment $d_{s}$, and the plasma obtains polarization $\mathfrak{P}=n_{s} d_{s}$, where $n_{s}$ is the density of the cell.

It is known [10], that the polarization of neighboring cells induces in the considered cell the electric field intensity

$$
E_{s}=\frac{4 \pi}{3} \mathfrak{P},
$$

and the cell obtains the energy

$$
\mathcal{E}_{s}=\frac{d_{s} E_{s}}{2} .
$$

The gravity force applied to the nucleus is proportional to its mass $A m_{p}$ (where $A$ is a mass number of the nucleus, $m_{p}$ is the proton mass). The cell consists of $Z$ electrons, the gravity force applied to the cell electron gas is proportional to $Z m_{e}$ (where $m_{e}$ is the electron mass). The difference of these forces tends to pull apart centers of positive and negative charges and to increase the dipole moment. The electric field $E_{s}$ resists it. The process obtains equilibrium at the balance of the arising electric force $\nabla \mathcal{E}_{s}$ and the difference of gravity forces applied to the electron gas and the nucleus:

$$
\nabla\left(\frac{2 \pi}{3} \frac{\mathfrak{P}^{2}}{n_{s}}\right)+\left(A m_{p}-Z m_{e}\right) \boldsymbol{g}=0
$$

Taking into account, that $\boldsymbol{g}=-\nabla \psi$, we obtain

$$
\frac{2 \pi}{3} \frac{\mathfrak{P}^{2}}{n_{s}}=\left(A m_{p}-Z m_{e}\right) \psi
$$

Hence,

$$
\mathfrak{P}^{2}=\frac{3 G M_{r}}{2 \pi r} n_{e}\left(\frac{A}{Z} m_{p}-m_{e}\right),
$$


where $\psi$ is the potential of the gravitational field, $n_{s}=\frac{n_{e}}{Z}$ is the density of cell (nuclei), $n_{e}$ is the density of the electron gas, $M_{r}$ is the mass of a star containing inside a sphere with radius $r$.

\subsection{The Equilibrium in Plasma Electron Gas Subsystem}

Nonuniformly polarized matter can be represented by an electric charge distribution with density [10]

$$
\tilde{\varrho}=\frac{\operatorname{div} E_{s}}{4 \pi}=\frac{\operatorname{div\mathfrak {P}}}{3} .
$$

The full electric charge of cells placed inside the sphere with radius $r$

$$
Q_{r}=4 \pi \int_{0}^{r} \tilde{\varrho} r^{2} \mathrm{~d} r
$$

determinants the electric field intensity applied to a cell placed on a distance $r$ from center of a star

$$
\tilde{\boldsymbol{E}}=\frac{Q_{r}}{r^{2}}
$$

As a result, the action of a nonuniformly polarized environment can be described by the force $\tilde{\varrho} \tilde{E}$. This force must be taken into account in the formulating of equilibrium equation. It leads to the following form of the Euler equation:

$$
\gamma \boldsymbol{g}+\tilde{\varrho} \tilde{\boldsymbol{E}}+\nabla P=0
$$

\section{The Internal Structure of a Star}

It was shown above that the state with the constant density is energetically favorable for a plasma at a very high temperature. The plasma in the central region of a star can possess by this property. The calculation made below shows that the mass of central region of a star with the constant density-the star core-is equal to $1 / 2$ of the full star mass. Its radius is approximately equal to $1 / 10$ of radius of a star, i.e. the core with high density take approximately $1 / 1000$ part of the full volume of a star. The other half of a stellar matter is distributed over the region placed above the core. It has a relatively small density and it could be called as a star atmosphere.

\subsection{The Plasma Equilibrium in the Star Core}

In this case, the equilibrium condition (Equation (28)) for the energetically favorable state of plasma with the steady density $n_{s}=$ const is achieved at

$$
\mathfrak{P}=\sqrt{G} \gamma_{\star} r,
$$

Here the mass density is $\gamma_{\star} \approx \frac{A}{Z} m_{p} n_{\star}$. The polarized state of the plasma can be described by a state with an electric charge at the density 


$$
\tilde{\rho}=\frac{1}{3} \operatorname{div} \mathfrak{P}=\sqrt{G} \gamma_{\star},
$$

and the electric field applied to a cell is

$$
\tilde{\boldsymbol{E}}=\frac{\boldsymbol{g}}{\sqrt{G}}
$$

As a result, the electric force applied to the cell will fully balance the gravity action

$$
\gamma \boldsymbol{g}+\tilde{\varrho} \tilde{\boldsymbol{E}}=0
$$

at the zero pressure gradient

$$
\nabla P=0
$$

\subsection{The Main Parameters of a Star Core (in Order of Values)}

At known density $n_{\star}$ of plasma into a core and its equilibrium temperature $\mathbb{T}_{\star}$, it is possible to estimate the mass $\mathbb{M}_{\star}$ of a star core and its radius $\mathbb{R}_{\star}$. In accordance with the virial theorem ${ }^{3}$, the kinetic energy of particles composing the steady system must be approximately equal to its potential energy with opposite sign:

$$
\frac{G \mathbb{M}_{\star}^{2}}{\mathbb{R}_{\star}} \approx k \mathbb{T}_{\star} \mathbb{N}_{\star}
$$

where $\mathbb{N}_{\star}=\frac{4 \pi}{3} \mathbb{R}_{\star}^{3} n_{\star}$ is full number of particle into the star core.

With using determinations derived above (21) and (25) derived before, we obtain

$$
\mathbb{M}_{\star} \approx \frac{\mathbb{M}_{C h}}{(A / Z)^{2}}
$$

where $\mathbb{M}_{C h}=\left(\frac{\hbar c}{G m_{p}^{2}}\right)^{3 / 2} m_{p}$ is the Chandrasekhar mass.

The radius of the core is approximately equal

$$
\mathbb{R}_{\star} \approx\left(\frac{\hbar c}{G m_{p}^{2}}\right)^{1 / 2} \frac{a_{B}}{Z(A / Z)} .
$$

where $A$ and $Z$ are the mass and the charge number of atomic nuclei the plasma consisting of.

\subsection{The Equilibrium State of the Plasma Inside the Star Atmosphere}

The star core is characterized by the constant mass density, the charge density, the temperature and the pressure. At a temperature typical for a star core, the plasma can be considered as ideal gas, as interactions between its particles are small in comparison with $k \mathbb{T}_{\star}$. In atmosphere, near surface of a star, the ${ }^{3}$ Below we shell use this theorem in its more exact formulation. 
temperature is approximately by $3 \div 4$ orders smaller. But the plasma density is lower. Accordingly, interparticle interaction is lower too and we can continue to consider this plasma as ideal gas.

In the absence of the gravitation, the equilibrium state of ideal gas in some volume comes with the pressure equalization, i.e. with the equalization of its temperature $T$ and its density $n$. This equilibrium state is characterized by the equalization of the chemical potential of the gas $\mu$ (Equation (11)).

\subsection{The Radial Dependence of Density and Temperature of Substance Inside a Star Atmosphere}

For the equilibrium system, where different parts have different temperatures, the following relation of the chemical potential of particles to its temperature holds ([7], \$25):

$$
\frac{\mu}{k T}=\text { const }
$$

As thermodynamic (statistical) part of chemical potential of monoatomic ideal gas is ([7], §45):

$$
\mu_{T}=k T \ln \left[\frac{n}{2}\left(\frac{2 \pi \hbar^{2}}{m k T}\right)^{3 / 2}\right],
$$

we can conclude that at the equilibrium

$$
n \sim T^{3 / 2} .
$$

In external fields the chemical potential of a gas ([7] \$25) is equal to

$$
\mu=\mu_{T}+\mathcal{E}^{\text {potential }}
$$

where $\mathcal{E}^{\text {potential }}$ is the potential energy of particles in the external field. Therefore in addition to fulfillment of condition Equation (45), in a field with Coulomb potential, the equilibrium needs a fulfillment of the condition

$$
-\frac{G M_{r} \gamma}{r k T_{r}}+\frac{\mathfrak{P}_{r}^{2}}{2 k T_{r}}=\text { const }
$$

(where $m$ is the particle mass, $M_{r}$ is the mass of a star inside a sphere with radius $r, \mathfrak{P}_{r}$ and $T_{r}$ are the polarization and the temperature on its surface. As on the core surface, the left part of Equation (47) vanishes, in the atmosphere

$$
M_{r} \sim r k T_{r} .
$$

Supposing that a decreasing of temperature inside the atmosphere is a power fuction with the exponent $x$, its value on a radius $r$ can be written as

$$
T_{r}=\mathbb{T}_{\star}\left(\frac{\mathbb{R}_{\star}}{r}\right)^{x}
$$

and in accordance with Equation (45), the density

$$
n_{r}=n_{\star}\left(\frac{\mathbb{R}_{\star}}{r}\right)^{3 x / 2} \text {. }
$$


At assumption that the powers of $r$ in the right and the left parts of condition Equation (48) are equal, we obtain $x=4$.

Thus, at using power dependencies for the description of radial dependencies of density and temperature, we obtain

$$
n_{r}=n_{\star}\left(\frac{\mathbb{R}_{\star}}{r}\right)^{6}
$$

and

$$
T_{r}=\mathbb{T}_{\star}\left(\frac{\mathbb{R}_{\star}}{r}\right)^{4}
$$

\subsection{The Mass of the Star Atmosphere and the Full Mass of a Star}

After integration of Equation (51), we can obtain the mass of the star atmosphere

$$
\mathbb{M}_{A}=4 \pi \int_{\mathbb{R}_{\star}}^{\mathbb{R}_{0}}(A / Z) m_{p} n_{\star}\left(\frac{\mathbb{R}_{\star}}{r}\right)^{6} r^{2} \mathrm{~d} r=\frac{4 \pi}{3}(A / Z) m_{p} n_{\star} \mathbb{R}_{\star}^{3}\left[1-\left(\frac{\mathbb{R}_{\star}}{\mathbb{R}_{0}}\right)^{3}\right]
$$

It is equal to its core mass

(to $\frac{\mathbb{R}_{\star}^{3}}{\mathbb{R}_{0}^{3}} \approx 10^{-3}$ ), where $\mathbb{R}_{0}$ is radius of a star.

Thus, the full mass of a star

$$
\mathbb{M}=\mathbb{M}_{A}+\mathbb{M}_{\star} \approx 2 \mathbb{M}_{\star}
$$

\subsection{The Energy of a Star}

The virial theorem ([7] [8]) is applicable to a system of particles if they have a finite moving into a volume $V$. If their interaction obeys to the Coulomb's law, their potential energy $\mathcal{E}^{\text {potential }}$, their kinetic energy $\mathcal{E}^{\text {kinetic }}$ and pressure $P$ are in the ratio:

$$
2 \mathcal{E}^{\text {kinetic }}+\mathcal{E}^{\text {potential }}=3 P V
$$

On the star surface, the pressure is absent and for the particle system as a whole:

$$
2 \mathcal{E}^{\text {kinetic }}=-\mathcal{E}^{\text {potential }}
$$

and the full energy of plasma particles into a star

$$
\mathcal{E}(\text { plasma })=\mathcal{E}^{\text {kinetic }}+\mathcal{E}^{\text {potential }}=-\mathcal{E}^{\text {kinetic }} .
$$

Let us calculate the separate items composing the full energy of a star.

\subsection{The Kinetic Energy of Plasma}

The kinetic energy of plasma into a core:

$$
\mathcal{E}_{\star}^{\text {kinitic }}=\frac{3}{2} k \mathbb{T}_{\star} \mathbb{N}_{\star} .
$$

The kinetic energy of atmosphere: 


$$
\mathcal{E}_{a}^{\text {kinetic }}=4 \pi \int_{\mathbb{R}_{\star}}^{\mathbb{R}_{0}} \frac{3}{2} k^{2} \mathbb{T}_{\star} n_{\star}\left(\frac{\mathbb{R}_{\star}}{r}\right)^{10} r^{2} \mathrm{~d} r \approx \frac{3}{7}\left(\frac{3}{2} k \mathbb{T}_{\star} \mathbb{N}_{\star}\right)
$$

The total kinetic energy of plasma particles

$$
\mathcal{E}^{\text {kinetic }}=\mathcal{E}_{\star}^{\text {kinetic }}+\mathcal{E}_{a}^{\text {kinetic }}=\frac{15}{7} k \mathbb{T}_{\star} \mathbb{N}_{\star}
$$

\subsection{The Potential Energy of Star Plasma}

Inside a star core, the gravity force is balanced by the force of electric nature. Correspondingly, the energy of electric polarization can be considered as balanced by the gravitational energy of plasma. As a result, the potential energy of a core can be considered as equal to zero.

In a star atmosphere, this balance is absent.

The gravitational energy of an atmosphere

$$
\mathcal{E}_{a}^{G}=-4 \pi G \mathbb{M}_{\star} \frac{A}{Z} m_{p} n_{\star} \int_{\mathbb{R}_{\star}}^{\mathbb{R}_{0}} \frac{1}{2}\left[2-\left(\frac{\mathbb{R}_{\star}}{r}\right)^{3}\right]\left(\frac{\mathbb{R}_{\star}}{r}\right)^{6} r \mathrm{~d} r
$$

or

$$
\mathcal{E}_{a}^{G}=\frac{3}{2}\left(\frac{1}{7}-\frac{1}{2}\right) \frac{G \mathbb{M}_{\star}^{2}}{\mathbb{R}_{\star}}=-\frac{15}{28} \frac{G \mathbb{M}_{\star}^{2}}{\mathbb{R}_{\star}}
$$

The electric energy of atmosphere is

$$
\mathcal{E}_{a}^{E}=-4 \pi \int_{\mathbb{R}_{\star}}^{R_{0}} \frac{1}{2} \rho \varphi r^{2} \mathrm{~d} r,
$$

where

$$
\tilde{\varrho}=\frac{1}{3 r^{2}} \frac{\mathrm{d} \mathfrak{P} r^{2}}{\mathrm{~d} r}
$$

and

$$
\tilde{\varphi}=\frac{4 \pi}{3} \mathfrak{P} r
$$

The electric energy:

$$
\mathcal{E}_{a}^{E}=-\frac{3}{28} \frac{G \mathbb{M}_{\star}^{2}}{\mathbb{R}_{\star}},
$$

and total potential energy of atmosphere:

$$
\mathcal{E}_{a}^{\text {potential }}=\mathcal{E}_{a}^{G}+\mathcal{E}_{a}^{E}=-\frac{9}{14} \frac{G \mathbb{M}_{\star}^{2}}{\mathbb{R}_{\star}} .
$$

The equilibrium in a star depends both on plasma energy and energy of radiation.

\subsection{The Temperature of a Star Core}

\subsubsection{The Energy of the Black Radiation}

The energy of black radiation inside a star core is 


$$
\mathcal{E}_{\star}(b r)=\frac{\pi^{2}}{15} k \mathbb{T}_{\star}\left(\frac{\mathbb{R}_{\star}}{\hbar c}\right)^{3} \mathbb{V}_{\star} .
$$

The energy of black radiation inside a star atmosphere is

$$
\mathcal{E}_{a}(b r)=4 \pi \int_{R_{\star}}^{R_{0}} \frac{\pi^{2}}{15} k \mathbb{T}_{\star}\left(\frac{k \mathbb{T}_{\star}}{\hbar c}\right)^{3}\left(\frac{\mathbb{R}_{\star}}{r}\right)^{16} r^{2} \mathrm{~d} r=\frac{3}{13} \frac{\pi^{2}}{15} k \mathbb{T}_{\star}\left(\frac{k \mathbb{T}_{\star}}{\hbar c}\right)^{3} \mathbb{V}_{\star} .
$$

The total energy of black radiation inside a star is

$$
\mathcal{E}(b r)=\mathcal{E}_{\star}(b r)+\mathcal{E}_{a}(b r)=\frac{16}{13} \frac{\pi^{2}}{15} k T_{\star}\left(\frac{k T_{\star}}{\hbar c}\right)^{3} V_{\star}=1.23 \frac{\pi^{2}}{15} k T_{\star}\left(\frac{k T_{\star}}{\hbar c}\right)^{3} V_{\star}
$$

\subsubsection{The Full Energy of a Star}

In accordance with Equation (57), the full energy of a star

$$
\mathcal{E}^{\text {star }}=-\mathcal{E}^{\text {kinetic }}+\mathcal{E}(b r)
$$

i.e.

$$
\mathcal{E}^{\mathrm{star}}=-\frac{15}{7} k \mathbb{T}_{\star} \mathbb{N}_{\star}+\frac{16}{13} \frac{\pi^{2}}{15} k \mathbb{T}_{\star}\left(\frac{k \mathbb{T}_{\star}}{\hbar c}\right)^{3} \mathbb{V}_{\star} .
$$

The steady state of a star is determined by a minimum of its full energy:

$$
\left(\frac{\mathrm{d} \mathcal{E}^{\mathrm{star}}}{\mathrm{d} \mathbb{T}_{\star}}\right)_{\mathbb{N}=\text { const }, \mathbb{V}=\text { const }}=0,
$$

it corresponds to the condition:

$$
-\frac{15}{7} \mathbb{N}_{\star}+\frac{64 \pi^{2}}{13 \times 15}\left(\frac{k \mathbb{T}_{\star}}{\hbar c}\right)^{3} \mathbb{V}_{\star}=0
$$

Together with Equation (21) it defines the equilibrium temperature of a star core:

$$
\mathbb{T}_{\star}=\left(\frac{25 \times 13}{28 \pi^{4}}\right)^{1 / 3}\left(\frac{\hbar c}{k a_{B}}\right) Z \approx Z \times 2.13 \times 10^{7} \mathrm{~K}
$$

\subsection{Main Parameters}

\subsubsection{The Star Mass}

The virial theorem connect kinetic energy of a system with its potential energy. In accordance with Equations (67) and (60)

$$
\frac{9}{14} \frac{G \mathbb{M}_{\star}^{2}}{\mathbb{R}_{\star}}=\frac{30}{7} k \mathbb{T}_{\star} \mathbb{N}_{\star} .
$$

Introducing the non-dimensional parameter

$$
\eta=\frac{G \mathbb{M}_{\star} \frac{A}{Z} m_{p}}{\mathbb{R}_{\star} k \mathbb{T}_{\star}},
$$

we obtain

$$
\eta=\frac{20}{3}=6.67
$$


and at taking into account Equations.(21) and (75), the core mass is

$$
\mathbb{M}_{\star}=\left[\frac{20}{3}\left(\frac{25 \times 13}{28}\right)^{1 / 3} \frac{3}{4 \times 3.14}\right]^{3 / 2} \frac{\mathbb{M}_{C h}}{\left(\frac{A}{Z}\right)^{2}}=6.84 \frac{\mathbb{M}_{C h}}{\left(\frac{A}{Z}\right)^{2}}
$$

The obtained equation plays a very important role, because together with Equation (54), it gives a possibility to predict the total mass of a star:

$$
\mathbb{M}=2 \mathbb{M}_{\star}=\frac{13.68 \mathbb{M}_{C h}}{\left(\frac{A}{Z}\right)^{2}} \approx \frac{25.34 \mathbb{M}_{\odot}}{\left(\frac{A}{Z}\right)^{2}} .
$$

The comparison of obtained prediction Equation (80) with measuring data gives a method to check our theory. Although there is no way to determine chemical composition of cores of far stars, some predictions can be made in this way. At first, there must be no stars which masses exceed the mass of the Sun by more than one and a half orders, because it accords to limiting mass of stars consisting from hydrogen with $A / Z=1$. Secondly, the action of a specific mechanism [6] can make neutron-excess nuclei stable, but it don't give a base to suppose that stars with $A / Z>10$ (and with mass in hundred times less than hydrogen stars) can exist. Thus, the theory predicts that the whole mass spectrum must be placed in the interval from 0.25 up to approximately 25 solar masses. These predications are verified by measurements quite exactly. The mass distribution of binary stars ${ }^{4}$ is shown in Figure 1 [11].

It is important, that the mass spectrum of binary stars (Figure 1) consists of series of well-isolated lines which are representing the stars with integer values of ratios $A / Z=3,4,5, \cdots$, corresponding hydrogen-3,4,5 ... or helium-6,8,10 $\ldots$ (also line with the half-integer ratio $A / Z=3 / 2$, corresponding, probably, to helium-3, Be-6, C-9...). The existence of stable stars with ratios $A / Z \geq 3$ raises questions. It is generally assumed that stars are composed of hydrogen-1, deuterium, helium-4 and other heavier elements with $A / Z \approx 2$. Nuclei with $A / Z \geq 3$ are the neutron-excess and so short-lived, that they can not build a long-lived stars. Neutron-excess nuclei can become stable under the action of mechanism of neutronization, which is acting inside the dwarfs. It is accepted to think that this mechanism must not work into the stars. The consideration of the effecting of the electron gas of a dense plasma on the nucleus is described in [6]. These calculations show that the electron gas of dense plasma should also lead to the neutronization mechanism and to the stabilization of the neutron-excess nuclei. This explains the existence of a stable of stars, where the plasma consists of nuclei with $A / Z \geq 3$.

At considering of (Figure 1), the question is arising: why there are so few stars, which are composed by very stable nuclei of helium-4? At the same time, there are many stars with $A / Z=4$, i.e. consisting apparently of a hydrogen- 4 , as well ${ }^{4}$ The use of these data is caused by the fact that only the measurement of parameters of binary star rotation gives a possibility to determine their masses with satisfactory accuracy. 


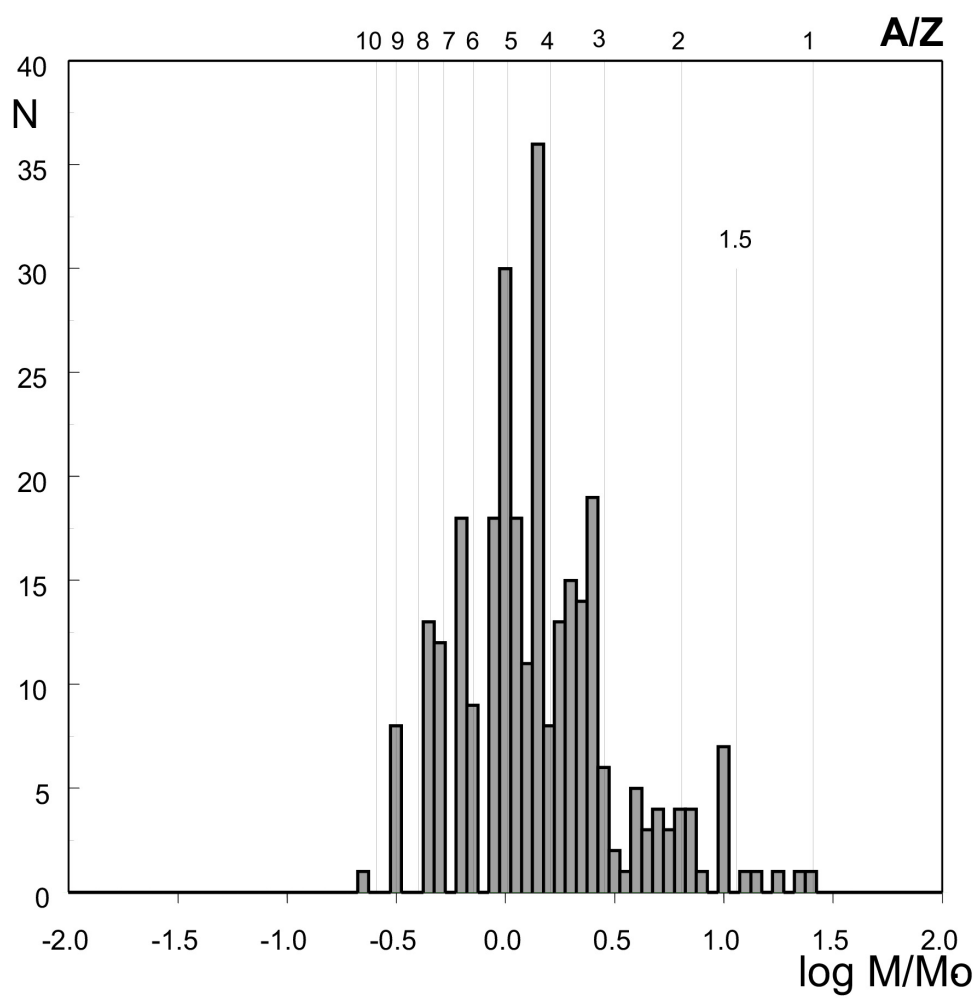

Figure 1. The mass distribution of binary stars [11]. On abscissa, the logarithm of the star mass over the Sun mass is shown. Solid lines mark masses, which agree with selected values of $\mathrm{A} / \mathrm{Z}$ from Equation (80).

as stars with $A / Z=3 / 2$, which hypothetically could be composed by another isotope of helium - helium-3. This equation is discussed in [6].

It is important to note, that according to Equation (80) the Sun must consist from a substance with $A / Z=5$. This conclusion is in a good agreement with results of consideration of solar oscillations [5].

\subsubsection{Radii of Stars}

Using Equation (21) and Equation (79), we can determine the star core radius:

$$
\mathbb{R}_{\star}=1.42 \frac{a_{B}}{Z(A / Z)}\left(\frac{\hbar c}{G m_{p}^{2}}\right)^{1 / 2} \approx \frac{9.79 \times 10^{10}}{Z(A / Z)} \mathrm{cm} .
$$

The temperature near the star surface is relatively small. It is approximately by 3 orders smaller than it is inside the core. Because of it at calculation of surface parameters, we must take into consideration effects of this order, i.e. it is necessary to take into account the gravity action on the electron gas. At that it is convenient to consider the plasma cell as some neutral quasi-atom (like the Thomas-Fermi atom). Its electron shell is formed by a cloud of free electrons.

Each such quasi-atom is retained on the star surface by its negative potential energy

$$
\left(\mathcal{E}_{\text {gravitational }}+\mathcal{E}_{\text {electric }}\right)<0
$$


The electron cloud of the cell is placed in the volume $\delta V=\frac{4 \pi}{3} r_{s}^{3}$, (where $r_{s} \approx\left(\frac{Z}{n_{e}}\right)^{1 / 3}$ ) under pressure $P_{e}$. The evaporation of plasma cell releases energy $\mathcal{E}_{P V}=P_{e} V_{s}$, and the balance equation takes the form:

$$
\mathcal{E}_{\text {gravitational }}+\mathcal{E}_{\text {electric }}+\mathcal{E}_{P V}=0 .
$$

In cold plasma, the electron cloud of the cell has energy $\mathcal{E}_{P V} \approx e^{2} n_{e}^{1 / 3}$. In very hot plasma at $k T \gg \frac{Z^{2} e^{2}}{r_{s}}$, this energy is equal to $\mathcal{E}_{P V}=\frac{3}{2} Z k T$. On the star surface these energies are approximately equal:

$$
\frac{k \mathbb{T}_{0}}{e^{2} n_{e}^{1 / 3}} \approx \frac{1}{\alpha}\left(\frac{\mathbb{R}_{0}}{\mathbb{R}_{\star}}\right)^{2} \approx 1
$$

One can show it easily, that in this case

$$
\mathcal{E}_{P V} \approx 2 Z \sqrt{\frac{3}{2} k T \cdot e^{2} n_{e}^{1 / 3}} .
$$

And if to take into account Equations (51)-(52), we obtain

$$
\mathcal{E}_{P V} \approx 1.5 Z k \mathbb{T}_{\star}\left(\frac{\mathbb{R}_{\star}}{\mathbb{R}_{0}}\right)^{3} \sqrt{\alpha \pi}
$$

The energy of interaction of a nucleus with its electron cloud does not change at evaporation of the cell and it can be neglected. Thus, for the surface

$$
\mathcal{E}_{\text {electric }}=\frac{2 \pi \mathfrak{P}^{2}}{3 n_{s}}=\frac{2 G \mathbb{M}_{\star}}{\mathbb{R}_{0}}\left(A m_{p}-Z m_{e}\right) .
$$

The gravitational energy of the cell on the surface

$$
\mathcal{E}_{\text {gravitational }}=-\frac{2 G \mathbb{M}_{\star}}{\mathbb{R}_{0}}\left(A m_{p}+Z m_{e}\right) .
$$

Thus, the balance condition Equation (83) on the star surface obtains the form

$$
-\frac{4 G \mathbb{M}_{\star} Z m_{e}}{\mathbb{R}_{0}}+1.5 Z k \mathbb{T}_{\star}\left(\frac{\mathbb{R}_{\star}}{\mathbb{R}_{0}}\right)^{3} \sqrt{\alpha \pi}=0 .
$$

\subsubsection{The $\mathbb{R}_{\star} / \mathbb{R}_{0}$ Ratio and $\mathbb{R}_{0}$}

With account of Equation (52) and Equations (78)-(77), we can write

$$
\frac{\mathbb{R}_{0}}{\mathbb{R}_{\star}}=\left(\frac{\sqrt{\alpha \pi}}{2 \eta} \frac{\frac{A}{Z} m_{p}}{m_{e}}\right)^{1 / 2} \approx 4.56 \sqrt{\frac{A}{Z}}
$$

As the star core radius is known Equation (81), we can obtain the star surface radius:

$$
\mathbb{R}_{0} \approx \frac{4.46 \times 10^{11}}{Z(A / Z)^{1 / 2}} \mathrm{~cm}
$$




\subsubsection{The Temperature of a Star Surface}

At known Equation (52) and Equation (75), we can calculate the temperature of external surface of a star

$$
\mathbb{T}_{0}=\mathbb{T}_{\star}\left(\frac{\mathbb{R}_{\star}}{\mathbb{R}_{0}}\right)^{4} \approx 4.92 \times 10^{5} \frac{Z}{(A / Z)^{2}}
$$

\subsubsection{The Comparison with Measuring Data}

The mass spectrum (Figure 1) shows that the Sun consists basically from plasma with $A / Z=5$. The radius of the Sun and its surface temperature are functions of $Z$ too. This values calculated at $A / Z=5$ and differen $Z$ are shown in Table 1.

One can see that these calculated data have a satisfactory agreement the measured radius of the Sun

$$
\mathbb{R}_{\odot}=6.96 \times 10^{10} \mathrm{~cm}
$$

and the measured surface temperature

$$
\mathbb{T}_{\odot}=5850 \mathrm{~K}
$$

at $Z=3$

The calculation shows that the mass of core of the Sun

$$
\mathbb{M}_{\star}(Z=3, A / Z=5) \approx 9.68 \times 10^{32} \mathrm{~g}
$$

i.t. almost exactly equals to one half of full mass of the Sun

$$
\frac{\mathbb{M}_{\star}(Z=3, A / Z=5)}{\mathbb{M}_{\odot}} \approx 0.486
$$

in full agreement with Equation (54).

In addition to obtained determinations of the mass of a star Equation (80), its temperature Equation (92) and its radius Equation (91) give possibility to check the calculation, if we compare these results with measuring data. Really, dependencies measured by astronomers can be described by functions:

$$
\begin{gathered}
\mathbb{M}=\frac{\text { Const }_{1}}{(A / Z)^{2}}, \\
\mathbb{R}_{0}=\frac{\text { Const }_{2}}{Z(A / Z)^{1 / 2}},
\end{gathered}
$$

Table 1. The calculated stellar parameters.

\begin{tabular}{ccc}
\hline$Z$ & $\begin{array}{c}\mathbb{R}_{\odot}, \mathrm{cm} \\
\text { (calculated (91)) }\end{array}$ & $\begin{array}{c}\mathbb{T}_{\odot}, \mathrm{K} \\
\text { (calculated (92)) }\end{array}$ \\
\hline 1 & $2.0 \times 10^{11}$ & 1961 \\
2 & $1.0 \times 10^{11}$ & 3923 \\
3 & $6.65 \times 10^{10}$ & 5885 \\
4 & $5.0 \times 10^{10}$ & 7845 \\
\hline
\end{tabular}




$$
\mathbb{T}_{0}=\frac{\text { Const }_{3} Z}{(A / Z)^{2}}
$$

If to combine they in the way, to exclude unknown parameter $Z$, one can obtain relation:

$$
\mathbb{T}_{0} \mathbb{R}_{0}=\text { Const } \mathbb{M}^{5 / 4}
$$

Its accuracy can by checked. For this checking, let us use the measuring data of parameters of masses, temperatures and radii of close binary stars [12] (see [6]). The results of these measurements are shown in Figure 2, where the dependence according to Equation (100). It is not difficult to see that these data are well described by the calculated dependence. It speaks about successfulness of our consideration.

\section{The Thermodynamic Relations of Intra-Stellar Plasma}

\subsection{The Thermodynamic Relation of Star Atmosphere Parameters}

Hot stars steadily generate energy and radiate it from their surfaces. This is non-equilibrium radiation in relation to a star. But it may be a stationary radiation for a star in steady state. Under this condition, the star substance can be considered as an equilibrium. This condition can be considered as quasi-adiabatic, because the interchange of energy between both subsystems-radiation and substance-is stationary and it does not result in a change of entropy of substance. Therefore, at consideration of state of a star atmosphere, one can base it on equilibrium conditions of hot plasma and the ideal gas law for adiabatic condition can be used for it in the first approximation.

It is known, that thermodynamics can help to establish correlation between steady-state parameters of a system. Usually, the thermodynamics considers systems at an equilibrium state with constant temperature, constant particle density and constant pressure over all system. The characteristic feature of the considered system is the existence of equilibrium at the absence of a constant temperature and particle density over atmosphere of a star. To solve this problem, one can introduce averaged pressure

$$
\hat{P} \approx \frac{G \mathbb{M}^{2}}{\mathbb{R}_{0}^{4}},
$$

averaged temperature

$$
\hat{T}=\frac{\int_{\mathbb{V}} T \mathrm{~d} V}{V} \sim \mathbb{T}_{0}\left(\frac{\mathbb{R}_{0}}{\mathbb{R}_{\star}}\right)
$$

and averaged particle density

$$
\hat{n} \approx \frac{\mathbb{N}_{A}}{\mathbb{R}_{0}^{3}}
$$

After it by means of thermodynamical methods, one can find relation between parameters of a star. 


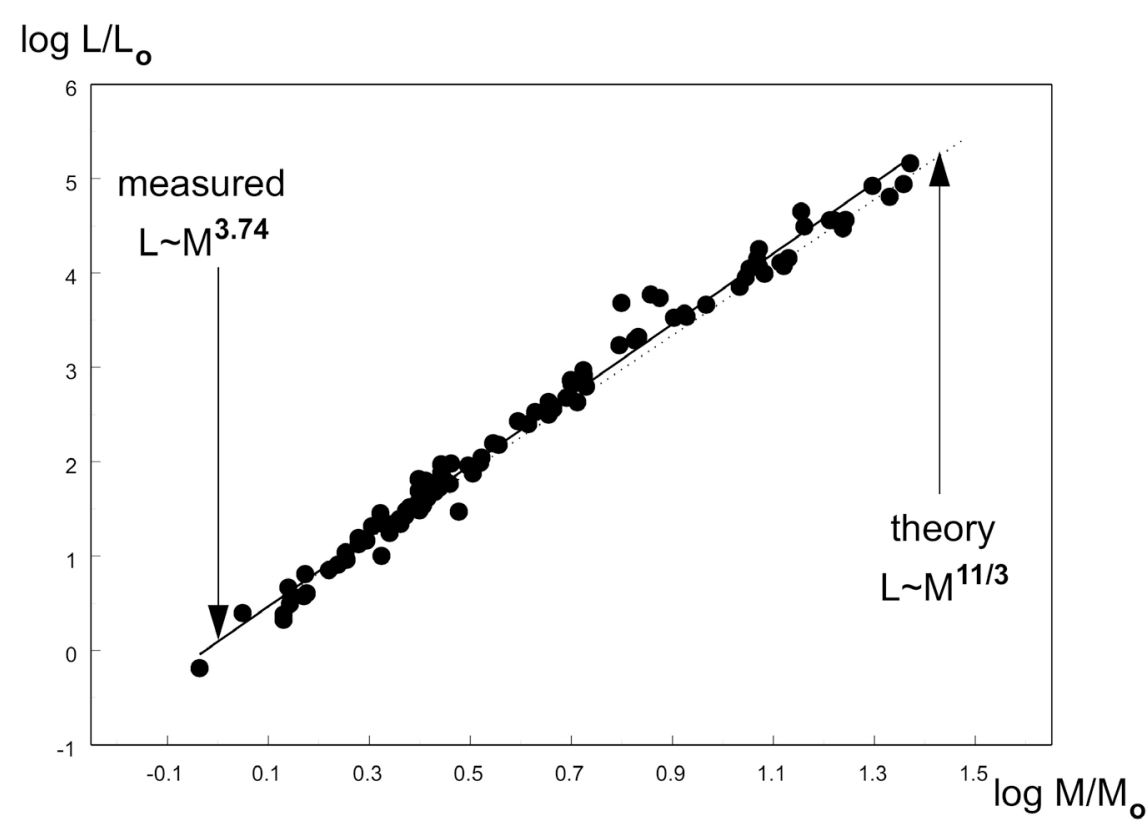

Figure 2. The relation between main parameters of stars (Equation (100)) and corresponding data of astronomical measurements for close binary stars are shown. These astronomical data were collected for the first time by K.F. Khaliullinin in his dissertation [12] (in Russian) and with his permission is republished in [6].

\subsubsection{The $c_{P} / c_{V}$ Ratio}

At a movement of particles according to the theorem of the equidistribution, the energy $k T / 2$ falls at each degree of freedom. It gives the heat capacity $c_{v}=3 / 2 k$.

According to the virial theorem [7] [8], the full energy of a star should be equal to its kinetic energy (with opposite sign), so as full energy related to one particle

$$
\mathcal{E}=-\frac{3}{2} k T
$$

In this case the heat capacity at constant volume (per particle over Boltzman's constant $k$ ) by definition is

$$
c_{V}=\left(\frac{\mathrm{d} E}{\mathrm{~d} T}\right)_{V}=-\frac{3}{2}
$$

The negative heat capacity of stellar substance is not surprising. It is a known fact and it is discussed in Landau-Lifshitz course [7]. The own heat capacity of each particle of star substance is positive. One obtains the negative heat capacity at taking into account the gravitational interaction between particles.

By definition the heat capacity of an ideal gas particle at permanent pressure [7] is

$$
c_{P}=\left(\frac{\mathrm{d} W}{\mathrm{~d} T}\right)_{P},
$$

where $W$ is enthalpy of a gas. 
As for the ideal gas [7]

$$
W-\mathcal{E}=N k T,
$$

and the difference between $c_{P}$ and $c_{V}$

$$
c_{P}-c_{V}=1 .
$$

Thus in the case considered, we have

$$
c_{P}=-\frac{1}{2} .
$$

Supposing that conditions are close to adiabatic ones, we can use the equation of the Poisson's adiabat.

\subsubsection{The Poisson's Adiabat}

The thermodynamical potential of a system consisting of $N$ molecules of ideal gas at temperature $T$ and pressure $P$ can be written as [7]:

$$
\Phi=\text { const } \cdot N+N T \ln P-N c_{P} T \ln T .
$$

The entropy of this system

$$
S=\text { const } \cdot N-N \ln P+N c_{P} \ln T .
$$

As at adiabatic process, the entropy remains constant

$$
-N T \ln P+N c_{P} T \ln T=\text { const, }
$$

we can write the equation for relation of averaged pressure in a system with its volume (The Poisson's adiabat) [7]:

$$
\hat{P} V^{\tilde{\gamma}}=\text { const, }
$$

where $\tilde{\gamma}=\frac{c_{P}}{c_{V}}$ is the exponent of adiabatic constant. In considered case taking into account of Equations.(106) and (105), we obtain

$$
\tilde{\gamma}=\frac{c_{P}}{c_{V}}=\frac{1}{3} .
$$

As $V^{1 / 3} \sim \mathbb{R}_{0}$, we have for equilibrium condition

$$
\hat{P} \mathbb{R}_{0}=\text { const. }
$$

\subsection{The Mass-Radius Ratio}

Using Equation (101) from Equation (115), we use the equation for dependence of masses of stars on their radii:

$$
\frac{\mathbb{M}^{2}}{\mathbb{R}_{0}^{3}}=\text { const }
$$

This equation shows the existence of internal constraint of chemical parameters of equilibrium state of a star. Indeed, the substitution of obtained determinations Equation (91) and (92)) into Equation (116) gives:

$$
Z \sim(A / Z)^{5 / 6}
$$

Simultaneously, the observational data of masses, of radii and their temperatures 
was obtained by astronomers for close binary stars [12]. The dependence of radii of these stars over these masses is shown in Figure 3 on double logarithmic scale. The solid line shows the result of fitting of measurement data $\mathbb{R}_{0} \sim \mathbb{M}^{0.68}$. It is close to theoretical dependence $\mathbb{R}_{0} \sim \mathbb{M}^{2 / 3}$ (Equation (116)) which is shown by dotted line.

If parameters of the star are expressed through corresponding solar values $\rho \equiv \frac{\mathbb{R}_{0}}{\mathbb{R}_{\odot}}$ and $\mu \equiv \frac{\mathbb{M}}{\mathbb{M}_{\odot}}$, that Equation (116) can be rewritten as

$$
\frac{\rho}{\mu^{2 / 3}}=1
$$

Numerical values of relations $\frac{\rho}{\mu^{2 / 3}}$ for close binary stars are obtained from Table [12] (see [6]).

\subsection{The Mass-Temperature and Mass-Luminosity Relations}

Taking into account Equations (52), (25) and (42) one can obtain the relation between surface temperature and the radius of a star

$$
\mathbb{T}_{0} \sim \mathbb{R}_{0}^{7 / 8}
$$

or accounting for Equation (116)

$$
\mathbb{T}_{0} \sim \mathbb{M}^{7 / 12}
$$

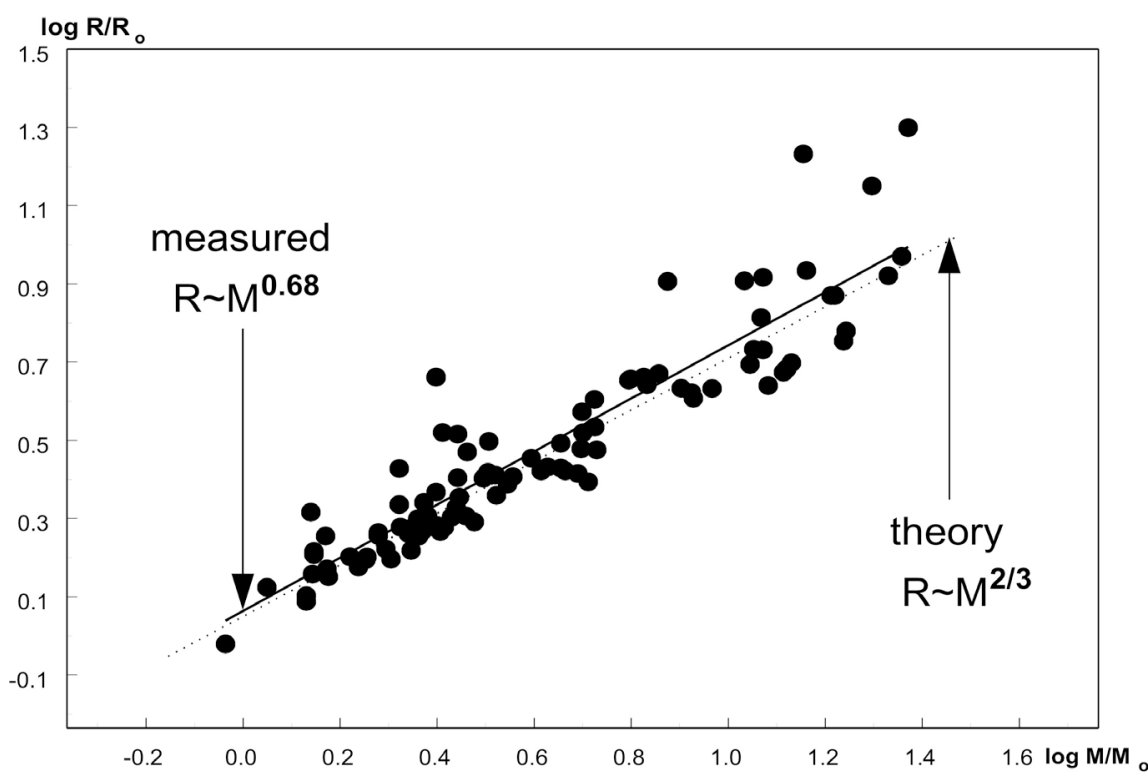

Figure 3. The dependence of radii of stars over the star mass. Here the radius of stars is normalized to the sunny radius, the stars masses are normalized to the mass of the Sum. The data are shown on double logarithmic scale. The solid line shows the result of fitting of measurement data $\mathbb{R}_{0} \sim \mathbb{M}^{0.68}$. The theoretical dependence $\mathbb{R}_{0} \sim \mathbb{M}^{2 / 3}$ (Equation (116)) is shown by the dotted line. These astronomical data were collected for the first time by K.F. Khaliullinin in his dissertation [12] (in Russian) and with his permission is republished in [6]. 
The dependence of the temperature on the star surface over the star mass of close binary stars [6] [12] is shown in Figure 4. Here the temperatures of stars are normalized to the sunny surface temperature $(5875 \mathrm{C})$, the stars masses are normalized to the mass of the Sum. The data are shown on double logarithmic scale. The solid line shows the result of fitting of measurement data $\left(\mathbb{T}_{0} \sim \mathbb{M}^{0.59}\right)$. The theoretical dependence $\mathbb{T}_{0} \sim \mathbb{M}^{7 / 12}$ (Equation (120)) is shown by dotted line.

If parameters of the star are expressed through corresponding solar values $\tau \equiv \frac{\mathbb{T}_{0}}{\mathbb{T}_{\odot}}$ and $\mu \equiv \frac{\mathbb{M}}{\mathbb{M}_{\odot}}$, that Equation (120) can be rewritten as

$$
\frac{\tau}{\mu^{7 / 12}}=1
$$

Numerical values of relations $\frac{\tau}{\mu^{7 / 12}}$ for close binary stars are shown in the last table of [6].

The analysis of these data leads to few conclusions. The averaging over all tabulated stars gives

$$
\left\langle\frac{\tau}{\mu^{7 / 12}}\right\rangle=1.007 \pm 0.07
$$

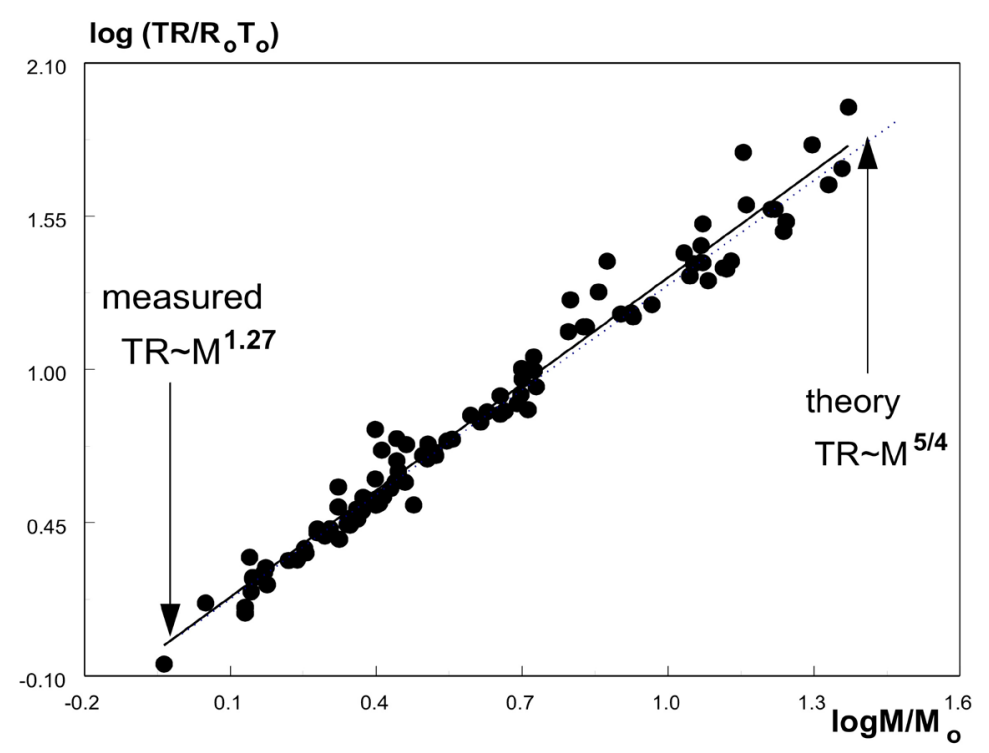

Figure 4. The dependence of the temperature on the star surface over the star mass of close binary stars. Here the temperatures of stars are normalized to surface temperature of the Sun $(5875 \mathrm{C})$, the stars masses are normalized to the mass of Sum. The data are shown on double logarithmic scale. The solid line shows the result of fitting of measurement data $\left(\mathbb{T}_{0} \sim \mathbb{M}^{0.59}\right)$. The theoretical dependence $\mathbb{T}_{0} \sim \mathbb{M}^{7 / 12}$ (Equation (120)) is shown by dotted line. These astronomical data were collected for the first time by K.F. Khaliullinin in his dissertation [12] (in Russian) and with his permission is republished in [6]. 
and we can conclude that the variability of measured data of surface temperatures and stellar masses has statistical character. Secondly, Equation (121) is valid for all hot stars (exactly for all close binary stars).

The problem with the averaging of $\frac{\rho}{\mu^{2 / 3}}$ looks different. There are a few of giants and super-giants in this Table. The values of ratio $\frac{\rho}{\mu^{2 / 3}}$ are more than 2 for them. It seems that, if to exclude these stars from consideration, the averaging over stars of the main sequence gives value close to 1 . Evidently, it needs in more detail consideration.

The luminosity of a star

$$
\mathbb{L}_{0} \sim \mathbb{R}_{0}^{2} \mathbb{T}_{0}^{4}
$$

at taking into account (Equation (116)) and (Equation (120)) can be expressed as

$$
\mathbb{L}_{0} \sim \mathbb{M}^{11 / 3} \sim \mathbb{M}^{3.67}
$$

This dependence is shown in Figure 5.

It can be seen that all calculated interdependencies $\mathbb{R}(\mathbb{M}), \mathbb{T}(\mathbb{M})$ and $\mathrm{L}(\mathbb{M})$ show a good qualitative agreement with the measuring data. At that it is important, that the quantitative explanation of mass-luminosity dependence discovered at the beginning of 20 th century is obtained.

\section{The Compilation of the Results of Calculations}

Let us put together the results of calculations. It is energetically favorable for the star to be divided into two volumes: the core is located in the central area of the star and the atmosphere is surrounding it from the outside (Figure 6).

The core has the radius:

$$
\mathbb{R}_{\star}=2.08 \frac{a_{B}}{Z(A / Z)}\left(\frac{\hbar c}{G m_{p}^{2}}\right)^{1 / 2} \approx \frac{1.41 \times 10^{11}}{Z(A / Z)} \mathrm{cm} .
$$

It is roughly equal to $1 / 10$ of the stellar radius.

At that the mass of the core is equal to

$$
\mathbb{M}_{\star}=6.84 \frac{\mathbb{M}_{C h}}{\left(\frac{A}{Z}\right)^{2}}
$$

It is almost exactly equal to one half of the full mass of the star.

The plasma inside the core has the constant density

$$
n_{\star}=\frac{16}{9 \pi} \frac{Z^{3}}{a_{B}^{3}} \approx 1.2 \times 10^{24} Z^{3} \mathrm{~cm}^{-3}
$$

and constant temperature

$$
\mathbb{T}_{\star}=\left(\frac{25 \times 13}{28 \pi^{4}}\right)^{1 / 3}\left(\frac{\hbar c}{k a_{B}}\right) Z \approx Z \times 2.13 \times 10^{7} \mathrm{~K} .
$$


$\log T / T_{0}$

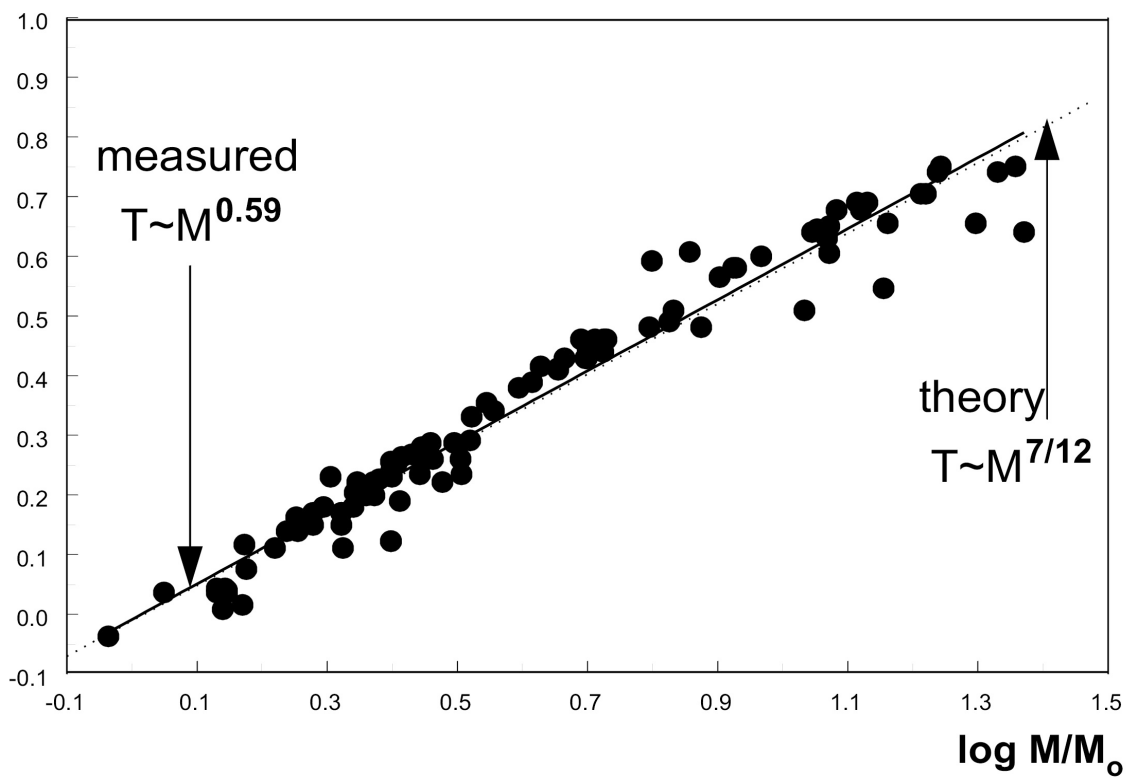

Figure 5. The dependence of star luminosity on the star mass of close binary stars. The luminosities are normalized to the luminosity of the Sun, the stars masses are normalized to the mass of the Sum. The data are shown on double logarithmic scale. The solid line shows the result of fitting of measurement data $\mathbb{L} \sim \mathbb{M}^{3.74}$. The theoretical dependence $\mathbb{L} \sim \mathbb{M}^{11 / 3}$ (Equation (124)) is shown by dotted line. These astronomical data were collected for the first time by K.F. Khaliullinin in his dissertation [12] (in Russian) and with his permission is republished in [6].

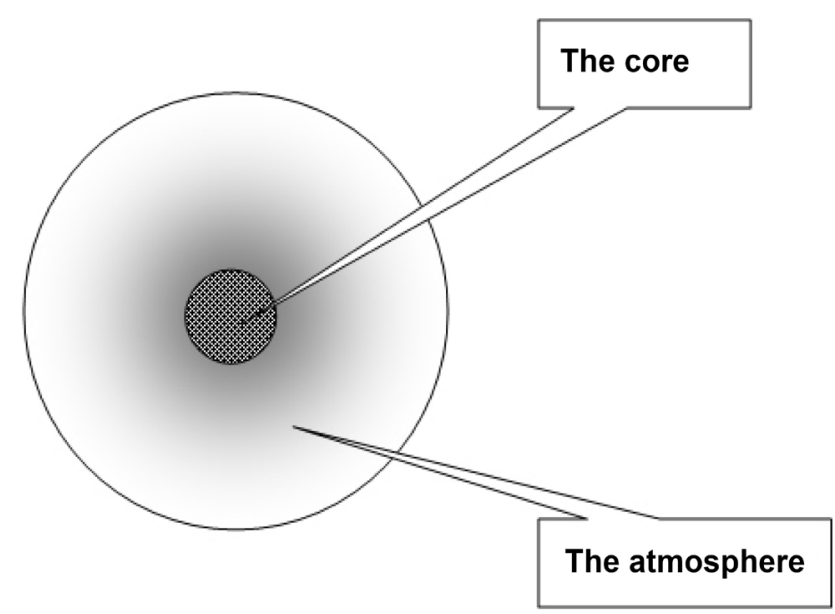

Figure 6. The schematic of the star interior.

The plasma density and its temperature are decreasing at an approaching to the stellar surface:

$$
n_{e}(r)=n_{\star}\left(\frac{\mathbb{R}_{\star}}{r}\right)^{6}
$$

and 


$$
T_{r}=\mathbb{T}_{\star}\left(\frac{\mathbb{R}_{\star}}{r}\right)^{4}
$$

The external radius of the star is determined as

$$
\mathbb{R}_{0}=\left(\frac{\sqrt{\alpha \pi}}{2 \eta} \frac{\frac{A}{Z} m_{p}}{m_{e}}\right)^{1 / 2} \mathbb{R}_{\star} \approx \frac{6.44 \times 10^{11}}{Z(A / Z)^{1 / 2}} \mathrm{~cm}
$$

and the temperature on the stellar surface is equal to

$$
\mathbb{T}_{0}=\mathbb{T}_{\star}\left(\frac{\mathbb{R}_{\star}}{\mathbb{R}_{0}}\right)^{4} \approx 4.92 \times 10^{5} \frac{Z}{(A / Z)^{2}}
$$

\subsection{About 《Black Holes》}

It seems that the idea about the 《black holes》 existence is organic related to the suggestion about an inevitable collapse of large cosmic bodies on the last stage of their evolutions. However, the models of collapsing masses were appearing as a consequence of the rejection from attention of the gravity induced electric polarization of the intra-stellar plasma. At a thermal energy lowering, the gravitational contraction of cooling star is balanced by a counteraction of electrically polarized plasma. If to take into account this mechanism, the possibility of collapse must be excludes.

It allows newly to take a look on the 《black hole》 problem.

In accordance with the standard approach, the Schwarzshield radius of 《black hole》 with mass $M_{b h}$ is

$$
r_{b h}=\frac{2 G M_{b h}}{c^{2}}
$$

and accordingly the average density of 《black holes》:

$$
\gamma_{b h}=\frac{3 c^{6}}{32 \pi M_{b h}^{2} G^{3}} \text {. }
$$

The estimations are showing that all large inwardly-galactic objects of all classes-stars, dwarves, pulsars, giants-possess the mass of the order $M_{C h}$ (or $\left.10 M_{C h}\right)$. As the density of these objects are small relatively to the limit Equation (134). As result, a searching of 《black hole》 inside stellar objects of our Galaxy seems as hopeless.

On the other hand, stellar objects, consisting of hot relativistic plasma-a quasars, in accordance with their mass and density, may stay 《black holes》. The process of collapse is not needed for their creation. As the quasar mass $M_{q u} \gg M_{C h}$, all other stellar objects must organize their moving around it and one can suppose that a 《black hole》 can exist at the center of our Galaxy.

\section{Conclusions}

Evidently, the main conclusion from the above consideration consists in statement of the fact that now there are quite enough measuring data to place 
the theoretical astrophysics on a reliable foundation. All above measuring data are known for a relatively long time. The traditional system of view based on the Euler equation in the form Equation (1) could not give a possibility to explain and even to consider with due proper attention to these data. Taking into account the gravity induced electric polarization of plasma and a change starting postulate gives a possibility to obtain results for explanation of measuring data considered above.

Basically these results are the following.

Using the standard method of plasma description leads to the conclusion that at conditions characteristic for the central stellar region, the plasma has the minimum energy at constant density $n_{\star}$ and at the constant temperature $\mathbb{T}_{\star}$.

This plasma forms the core of a star, where the pressure is constant and gravity action is balanced by the force of the gravity induced by the electric polarization. The virial theorem gives a possibility to calculate the stellar core mass $\mathbb{M}_{\star}$ and its radius $\mathbb{R}_{\star}$. At that the stellar core volume is approximately equal to $1 / 1000$ part of full volume of a star.

The remaining mass of a star located over the core has a density approximately thousand times smaller and it is convenient to name it a star atmosphere. At using thermodynamical arguments, it is possible to obtain the radial dependence of plasma density inside the atmosphere $n_{a} \approx r^{-6}$ and the radial dependence of its temperature $\mathbb{T}_{a} \approx r^{-4}$.

It gives a possibility to conclude that the mass of the stellar atmosphere $\mathbb{M}_{a}$ is almost exactly equal to the stellar core mass. Thus, the full stellar mass can be calculated. It depends on the ratio of the mass and the charge of nuclei composing the plasma. This claim is in a good agreement with the measuring data of the mass distribution of binary stars (and close binary stars too). ${ }^{5}$ At that it is important that the upper limit of masses of both binary stars and close binary stars is in accordance with the calculated value of the mass of the hydrogen star. The obtained formula explains the origin of sharp peaks of stellar mass distribution. They evidence that the substance of these stars has a certain value of the ratio $A / Z$. In particular, the solar plasma consists of nuclei with $A / Z=5$.

Knowing temperature and substance density on the core and knowing their radial dependencies, it is possible to estimate the surface temperature $\mathbb{T}_{0}$ and the radius of a star $\mathbb{R}_{0}$. It turns out that these measured parameters must be related to the star mass with the ratio $\mathbb{T}_{0} \mathbb{R}_{0} \sim \mathbb{M}^{5 / 4}$. It is in a good agreement with measuring data.

Using another thermodynamical relation-the Poisson's adiabat-gives a way to determine the relation between radii of stars and their masses $\mathbb{R}_{0}^{3} \sim \mathbb{M}^{2}$, and between their surface temperatures and masses $\mathbb{T}_{0} \sim \mathbb{M}^{5 / 7}$. It gives the quantitative explanation of the mass-luminosity dependence.

The model described above gives a quite satisfactory quantitative explanation ${ }^{5}$ The measurement of parameters of these stars has a satisfactory accuracy only. 
to other astronomical data, such as seismic oscillations of the solar surface, magnetic fields of stars, etc. [5].

The concept of star evolution, including their collapse and "black hole" formation, is flawed.

\section{Conflicts of Interest}

The author declares no conflicts of interest regarding the publication of this paper.

\section{References}

[1] Vasiliev, B.V. (2001) Nuovo Cimento B, 116, 617-634.

[2] Vasiliev, B.V. (1997) Nuovo Cimento B, 112, 1361-1372.

[3] Vasiliev, B.V. (1996) Nuovo Cimento B, 110, 381-389.

[4] Vasiliev, B. (2014) Universal Journal of Physics and Application, 2, 257-262.

[5] Vasiliev, B. (2014) Universal Journal of Physics and Application, 2, 284-301.

[6] Vasiliev, B. (2014) Universal Journal of Physics and Application, 2, 228-243.

[7] Landau, L.D. and Lifshits, E.M. (1980) Statistical Physics. 3rd Edition, Vol. 1, Pergamon, Oxford.

[8] Vasiliev, B.V. and Luboshits, V.L. (1994) Physics-Uspekhi, 37, 345. https://doi.org/10.1070/PU1994v037n04ABEH000018

[9] Leung, Y.C. (1984) Physics of Dense Matter. Science Press/World Scientific, Beijing and Singapore.

[10] Landau, L.D. and Lifshits, E.M. (1980) Electrodynamics of Condensed Matter. 3rd Edition, Vol. 1, Pergamon, Oxford.

[11] Heintz, W.D. (1978) Double Stars. In: Geophysics and Astrophysics Monographs, Vol. 15, Springer, Dordrecht. https://doi.org/10.1007/978-94-009-9836-0

[12] Khaliullin, K.F. (2004) Dissertation. Sternberg Astronomical Institute, Moscow. 